\title{
Parity of the neutron consistent with neutron-antineutron oscillations
}

\author{
Kazuo Fujikawa ${ }^{1}$ and Anca Tureanu $\odot^{2}$ \\ ${ }^{1}$ Interdisciplinary Theoretical and Mathematical Sciences Program, RIKEN, Wako 351-0198, Japan \\ ${ }^{2}$ Department of Physics, University of Helsinki, P.O. Box 64, FIN-00014 Helsinki, Finland
}

(Received 25 October 2020; accepted 21 February 2021; published 30 March 2021)

\begin{abstract}
In the analysis of neutron-antineutron oscillations, it has recently been argued in the literature that the use of the $i \gamma^{0}$ parity $n^{p}(t,-\vec{x})=i \gamma^{0} n(t,-\vec{x})$, which is consistent with the Majorana condition, is mandatory and that the ordinary parity transformation of the neutron field $n^{p}(t,-\vec{x})=\gamma^{0} n(t,-\vec{x})$ has difficulties. We show that a careful treatment of the ordinary parity transformation of the neutron works in the analysis of neutron-antineutron oscillations. Technically, $C P$ symmetry in a mass diagonalization procedure is important and the two parity transformations $i \gamma_{0}$-parity and $\gamma_{0}$-parity are compensated for by the PauliGürsey transformation. Our analysis shows that either choice of parity gives the correct results for neutronantineutron oscillations if carefully treated.
\end{abstract}

DOI: 10.1103/PhysRevD.103.065017

\section{INTRODUCTION}

Motivated by the possible baryon number violation in some unification schemes, many authors have discussed neutron-antineutron oscillations in the past [1-9] (see also Refs. $[10,11])$ and, in spite of the phenomenon not yet having been observed, experimental bounds have been established [12]. Experiments are planned to continue in the near future [13]. Interest in the theoretical aspects of discrete symmetries in the context of neutron-antineutron oscillations was recently stimulated by Ref. [14], which was then followed by several related works [15-21].

Historically, it appears that researchers did not pay much attention to the existence of different definitions of the parity operation or simply used conventional $\gamma^{0}$-parity in analyses of neutron oscillations. In [18], for example, two definitions of parity were used in the analysis of two different aspects of neutron oscillations and $i \gamma^{0}$-parity was used in [19]. Recently, Berezhiani and Vainshtein [21] performed a detailed analysis of neutron-antineutron oscillations using a two-component spinor notation and $i \gamma^{0}$ parity. They showed consistency in the use of $i \gamma^{0}$-parity in their analysis of neutron-antineutron oscillations. They also commented that ordinary $\gamma^{0}$-parity presents difficulties in analyses of neutron oscillations. In this paper, however, we will show that the $\gamma^{0}$-parity for the initial neutron field is perfectly consistent if the neutron oscillations are properly

Published by the American Physical Society under the terms of the Creative Commons Attribution 4.0 International license. Further distribution of this work must maintain attribution to the author(s) and the published article's title, journal citation, and DOI. Funded by SCOAP ${ }^{3}$. formulated using $C P$ symmetry for the characterization of the emergent Majorana fermions. Our analysis justifies the common use of $\gamma^{0}$-parity in neutron-antineutron oscillations in the past. Combined with the analysis of $i \gamma^{0}$-parity in [21], one can thus use either definition of parity in an analysis of neutron oscillations. We emphasize that the known physics related to the neutron-namely, the hadron scattering and the entirety nuclear physics, is based on the use of $\gamma^{0}$-parity (i.e., intrinsic parities of neutron and antineutron \pm 1 ). Thus the consistent description of neutron-antineutron oscillations by $\gamma^{0}$-parity is in fact gratifying.

To fix the ideas and conventions, we start from the quadratic effective Hermitian Lagrangian for the neutron field $n(x)$ with general small $\Delta B=2$ terms added:

$$
\begin{aligned}
\mathcal{L}= & \bar{n}(x) i \gamma^{\mu} \partial_{\mu} n(x)-m_{D} \bar{n}(x) n(x) \\
& -\frac{1}{2}\left[m n^{T}(x) C n(x)+m^{\dagger} \bar{n}(x) C \bar{n}^{T}(x)\right] \\
& -\frac{1}{2}\left[m_{5} n^{T}(x) C \gamma_{5} n(x)-m_{5}^{\dagger} \bar{n}(x) C \gamma_{5} \bar{n}^{T}(x)\right],
\end{aligned}
$$

where $m_{D}$ is chosen to be a real positive parameter and $m$ and $m_{5}$ are complex parameters, very small in absolute value, which break the baryon number symmetry. Our notational conventions follow [22]; in particular, the charge conjugation matrix is defined by $C=i \gamma^{2} \gamma^{0}$. The parity-violating and fermion number preserving term $\overline{n(x)}\left(i \gamma_{5} \delta m\right) n(x)$ is eliminated by a chiral transformation within the framework of Eq. (1). We analyze the full implications of Eq. (1) without any further constraints, except for the assumption that the magnitudes of $|m|$ and $\left|m_{5}\right|$ are very small compared to the neutron mass $m_{D}$. It is 
known [10,11] that the main aspects of the possible neutron-antineutron oscillations are described by the above Lagrangian.

We define the basic discrete transformation operations based on the free neutron which is assumed to be a Dirac field:

$$
\int d^{4} x \mathcal{L}_{D}=\int d^{4} x\left\{\bar{n}(x) i \gamma^{\mu} \partial_{\mu} n(x)-m_{D} \bar{n}(x) n(x)\right\}
$$

We define the charge conjugation $C$, which is given by the representation theory of the Clifford algebra, by

$$
n(x) \rightarrow n^{c}(x)=C \overline{n(x)}^{T}, \quad n^{c}(x) \rightarrow n(x),
$$

and the parity $P$ is defined as the mirror symmetry for a Dirac fermion by the customarily used " $\gamma$-parity"

$$
n(t, \vec{x}) \rightarrow n^{p}(t,-\vec{x})=\gamma^{0} n(t,-\vec{x})
$$

Both $C$ and $P$, thus defined, preserve the Dirac Lagrangian (2) invariant. The $C P$ transformation rules are defined by

$$
\begin{aligned}
n(t, \vec{x}) \rightarrow \mathcal{P C} n(t, \vec{x}) \mathcal{C}^{\dagger} \mathcal{P}^{\dagger} & =n^{c p}(t,-\vec{x})=\left(n^{c}\right)^{p}(t,-\vec{x}) \\
& =-\gamma^{0} n^{c}(t,-\vec{x}), \\
n(t, \vec{x}) \rightarrow \mathcal{C P} n(t, \vec{x}) \mathcal{P}^{\dagger} \mathcal{C}^{\dagger} & =n^{p c}(t,-\vec{x})=\left(n^{p}\right)^{c}(t,-\vec{x}) \\
& =\gamma^{0} n^{c}(t,-\vec{x}),
\end{aligned}
$$

where we use $\left(n^{c}\right)^{p}(t,-\vec{x})=C \bar{\gamma}^{0} n(t,-\vec{x})^{T}$. Thus the ordering is important, but when operating on fermionic fields in a general Lagrangian, which is quadratic in fermions, the ordering is not important. The parity transformation of the charge conjugated fields is

$$
n^{c}(x) \rightarrow\left(n^{c}\right)^{p}(t,-\vec{x})=-\gamma^{0} n^{c}(t,-\vec{x}) .
$$

This definition of parity amounts to assigning an intrinsic parity +1 to the neutron and -1 to the antineutron.

On the other hand, in the original work of Majorana [23], the free Majorana fermion was defined by the same action as the Dirac fermion in Eq. (2) but with purely imaginary Dirac gamma matrices $\gamma^{\mu}$. Then the free Dirac equation

$$
\left[i \gamma^{\mu} \partial_{\mu}-m\right] \psi(x)=0
$$

is a real differential equation, and one can impose the reality condition on the solution

$$
\psi(x)^{\star}=\psi(x),
$$

which implies the self-conjugation property under the charge conjugation. ${ }^{1}$ The conventional parity transformation $\psi(x) \rightarrow \psi^{p}(t,-\vec{x})=\gamma^{0} \psi(t,-\vec{x})$ cannot maintain the reality condition (8) for the purely imaginary $\gamma^{0}$. Thus the "i $\gamma^{0}$-parity"

$$
\psi(x) \rightarrow \psi^{p}(t,-\vec{x})=i \gamma^{0} \psi(t,-\vec{x})
$$

is chosen as a natural parity transformation rule for the Majorana fields $[23,24]$.

In a generic representation of the Dirac matrices [22], the $i \gamma^{0}$-parity satisfies the condition

$$
i \gamma^{0} \psi(t,-\vec{x})=C{\overline{i \gamma^{0} \psi(t,-\vec{x}}}^{T}
$$

for the field which satisfies the classical Majorana condition

$$
\psi(x)=C \overline{\psi(x)}^{T},
$$

and thus $i \gamma^{0}$-parity is also a natural choice for the parity of the Majorana fermion in this generic representation (as well as in any other). See [25] for the phase freedom in the definition of the parity operation. $i \gamma^{0}$-parity can be used for free Dirac fields as well, with the comment that the intrinsic parity assigned to the corresponding particle and antiparticle is the same, $i$.

For the Dirac fermion with $U(1)$ fermion number freedom, these two definitions of parity are equivalent, but their equivalence is not obvious for theories with broken fermionic number such as Eq. (1). One may suspect that the conventional $\gamma^{0}$-parity is inconsistent in theories where Majorana fermions appear. In the analysis of neutronantineutron oscillations described by Eq. (1), one visualizes a virtual process where the initial neutron turns into a superposition of nondegenerate Majorana fermions which after oscillation ends up being an antineutron. If one uses the $\gamma^{0}$-parity operation for the starting neutron, one may thus suspect that a contradiction appears for the intermediate states with Majorana fermions. On the other hand, $i \gamma^{0}$-parity is consistent with both Dirac and Majorana fermions and thus intuitively more natural for neutronantineutron oscillations.

In this paper, however, we will show that the use of the conventional $\gamma^{0}$-parity for the starting neutron field gives a logically consistent description of neutron-antineutron oscillations if a proper treatment and interpretation is applied. The basic idea leading to this statement is that $C$ and $P$ defined for the free Dirac Lagrangian (2) described above are not generally well defined after the mass diagonalization of the general Lagrangian (1) (see, however,

\footnotetext{
${ }^{1}$ The pure imaginary condition $\psi^{\star}(x)=-\psi(x)$ is also an allowed solution, but we take Eq. (8) as the primary definition in this paper.
} 
Appendix A), but the $C P$ symmetry is defined for the general Lagrangian (1) after the diagonalization of the mass terms; the mass diagonalization is, after all, required to define Majorana fermions. The proposal in [26] is then to characterize the emergent Majorana fermions by $C P$ symmetry. ${ }^{2}$ A formal proof of the canonical equivalence of the two choices of parity operation in the analysis of neutron-antineutron oscillations will be given in Appendix A using the Pauli-Gürsey transformation.

\section{NEUTRON-ANTINEUTRON OSCILLATIONS WITH $\gamma^{0}$-PARITY}

\section{A. Consistent description of Majorana fermions}

We first rewrite the Hermitian Lagrangian (1) in terms of chiral notations as

$$
\begin{aligned}
\mathcal{L} & =\overline{n_{L}}(x) i \gamma^{\mu} \partial_{\mu} n_{L}(x)+\overline{n_{R}}(x) i \gamma^{\mu} \partial_{\mu} n_{R}(x)-m_{D} \overline{n_{L}}(x) n_{R}(x) \\
& -\frac{1}{2} m_{L} n_{L}^{T}(x) C n_{L}(x)-\frac{1}{2} m_{R} n_{R}^{T}(x) C n_{R}(x)+\text { H.c. },
\end{aligned}
$$

with $n_{R, L}(x)=\left[\left(1 \pm \gamma_{5}\right) / 2\right] n(x)$, for an effective use of the $C P$ transformation to characterize the Majorana fermions. In terms of the mass parameters in Eq. (1),

$$
m_{D}, \quad m_{L}=m-m_{5}, \quad m_{R}=m+m_{5} .
$$

Namely, we define the complex mass parameters $m_{L}$ and $m_{R}$, while $m_{D}$ is chosen to be real.

We recall the transformation laws of chiral fermions derived from the chiral projection of the Dirac fermionic field:

$$
\begin{aligned}
C: n_{L}(x) & \rightarrow C{\overline{n_{R}(x)}}^{T}, \quad n_{R}(x) \rightarrow C{\overline{n_{L}(x)}}^{T}, \\
P: n_{L}(x) & \rightarrow \gamma^{0} n_{R}(t,-\vec{x}), \quad n_{R}(x) \rightarrow \gamma^{0} n_{L}(t,-\vec{x}), \\
C P: n_{L}(x) & \rightarrow-\gamma^{0} C{\overline{n_{L}(t,-\vec{x}}}^{T}, \quad n_{R}(x) \rightarrow-\gamma^{0} C{\overline{n_{R}(t,-\vec{x}}}^{T} .
\end{aligned}
$$

The minus signature in the $C P$ transformation shows that we are using the $n^{c p}(t,-\vec{x})$ convention from Eq. (5). The baryon-number-violating mass terms in the Lagarangian (12) are transformed under $C, P$, and $C P$ transformations in Eq. (14) as

\footnotetext{
${ }^{2}$ It has been recently shown [27] that the use of either $\gamma^{0}$-parity or $i \gamma^{0}$-parity for chiral fermions gives a consistent equivalent description of emergent Majorana fermions in Weinberg's model of neutrinos [28] in an extension of the Standard Model when $C P$ is used to characterize the Majorana neutrino formed of chiral fermions.
}

$$
\begin{aligned}
C: & -\frac{1}{2} m_{L} n_{L}^{T}(x) C n_{L}(x)-\frac{1}{2} m_{R} n_{R}^{T}(x) C n_{R}(x)+\text { H.c. } \\
& \rightarrow-\frac{1}{2} m_{R}^{\dagger} n_{L}^{T}(x) C n_{L}(x)-\frac{1}{2} m_{L}^{\dagger} n_{R}^{T}(x) C n_{R}(x)+\text { H.c. } \\
P: & -\frac{1}{2} m_{L} n_{L}^{T}(x) C n_{L}(x)-\frac{1}{2} m_{R} n_{R}^{T}(x) C n_{R}(x)+\text { H.c. } \\
& \rightarrow+\frac{1}{2} m_{L} n_{R}^{T}(x) C n_{R}(x)+\frac{1}{2} m_{R} n_{L}^{T}(x) C n_{L}(x)+\text { H.c. }, \\
C P: & -\frac{1}{2} m_{L} n_{L}^{T}(x) C n_{L}(x)-\frac{1}{2} m_{R} n_{R}^{T}(x) C n_{R}(x)+\text { H.c. } \\
& \rightarrow+\frac{1}{2} m_{L}^{\dagger} n_{L}^{T}(x) C n_{L}(x)+\frac{1}{2} m_{R}^{\dagger} n_{R}^{T}(x) C n_{R}(x)+\text { H.c. }
\end{aligned}
$$

Namely, in the Lagrangian (12), $C$ is a good symmetry for $m_{L}=m_{R}^{\dagger}$ and $P$ is a good symmetry for $m_{L}=-m_{R} ; C P$ is a good symmetry for

$$
m_{L}=-m_{L}^{\dagger} \quad \text { and } \quad m_{R}=-m_{R}^{\dagger},
$$

which can hold without any relation between $m_{L}$ and $m_{R}$. We emphasize, however, that these symmetry properties of the "bare parameters" have no definite meaning after the mass diagonalization in general, since the canonical transformation parametrized by the matrix $U$ which diagonalizes the mass terms to define the Majorana fermions carries away these discrete symmetries from the sector of the fermion mass terms to the interaction terms, as in the Standard Model. The actual discrete symmetries are better analyzed based on the Lagrangian after the mass diagonalization. On the other hand, one may want to know the more detailed direct physical implications of the starting Lagrangian based on direct diagonalization. This aspect is briefly mentioned in Appendix B.

We next diagonalize the Lagrangian (12) by writing the mass term as

$(-2) \mathcal{L}_{\text {mass }}=\left(\begin{array}{ll}\overline{n_{R}} & \overline{n_{R}^{c}}\end{array}\right)\left(\begin{array}{ll}m_{R}^{\dagger} & m_{D} \\ m_{D} & m_{L}\end{array}\right)\left(\begin{array}{c}n_{L}^{c} \\ n_{L}\end{array}\right)+$ H.c.,

where we define

$$
n_{L}^{c} \equiv C{\overline{n_{R}}}^{T}, \quad n_{R}^{c} \equiv C{\overline{n_{L}}}^{T} .
$$

We diagonalize the complex symmetric mass matrix using a $2 \times 2$ unitary matrix (Autonne-Takagi factorization) $[29,30]$

$$
U^{T}\left(\begin{array}{cc}
m_{R}^{\dagger} & m_{D} \\
m_{D} & m_{L}
\end{array}\right) U=i\left(\begin{array}{cc}
M_{1} & 0 \\
0 & M_{2}
\end{array}\right)
$$


where $M_{1}$ and $M_{2}$ are positive real numbers which can be chosen as characteristic values. ${ }^{3}$ This form of diagonalization makes the Lagrangian with diagonal mass matrix $C P$ invariant.

When one defines

$$
\left(\begin{array}{l}
n_{L}^{c} \\
n_{L}
\end{array}\right)=U\left(\begin{array}{c}
N_{L}^{c} \\
N_{L}
\end{array}\right), \quad\left(\begin{array}{l}
n_{R} \\
n_{R}^{c}
\end{array}\right)=U^{\star}\left(\begin{array}{c}
N_{R} \\
N_{R}^{c}
\end{array}\right)
$$

the mass term of the Lagrangian (12) becomes

$$
(-2) \mathcal{L}_{\text {mass }}=i\left(\begin{array}{ll}
\overline{N_{R}} & \overline{N_{R}^{c}}
\end{array}\right)\left(\begin{array}{cc}
M_{1} & 0 \\
0 & M_{2}
\end{array}\right)\left(\begin{array}{c}
N_{L}^{c} \\
N_{L}
\end{array}\right)+\text { H.c. }
$$

$$
\begin{aligned}
\mathcal{L} & =\frac{1}{2}\left\{\overline{N_{L}}(x) i \not \partial N_{L}(x)+\overline{N_{L}^{c}}(x) i \not \partial N_{L}^{c}(x)+\overline{N_{R}}(x) i \not \partial N_{R}(x)+\overline{N_{R}^{c}}(x) i \not \partial N_{R}^{c}(x)\right\}-(i / 2)\left(\overline{N_{R}} \quad \overline{N_{R}^{c}}\right)\left(\begin{array}{cc}
M_{1} & 0 \\
0 & M_{2}
\end{array}\right)\left(\begin{array}{c}
N_{L}^{c} \\
N_{L}
\end{array}\right)+\text { H.c. } \\
& =\overline{N_{L}}(x) i \not \partial N_{L}(x)+\overline{N_{R}}(x) i \not \partial N_{R}(x)-(i / 2)\left\{\overline{N_{R}} C M_{1}{\overline{n_{R}}}^{T}-N_{R}^{T} C M_{1} N_{R}-\overline{N_{L}} C M_{2} \overline{N_{L}}+N_{L}^{T} C M_{2} N_{L}\right\} \\
& =\frac{1}{2} \overline{\psi_{+}(x)}\left(i \gamma^{\mu} \partial_{\mu}-M_{1}\right) \psi_{+}(x)+\frac{1}{2} \overline{\psi_{-}(x)}\left(i \gamma^{\mu} \partial_{\mu}-M_{2}\right) \psi_{-}(x)
\end{aligned}
$$

where we define the Majorana fields by

$$
\begin{aligned}
& \psi_{+}(x)=e^{i \pi / 4} N_{R}(x)-e^{-i \pi / 4} C \overline{N_{R}(x)}{ }^{T}, \\
& \psi_{-}(x)=e^{i \pi / 4} N_{L}(x)+e^{-i \pi / 4} C \overline{N_{L}(x)},
\end{aligned}
$$

which satisfy the classical Majorana conditions

$$
\psi_{+}(x)=-C \overline{\psi_{+}(x)}{ }^{T}, \quad \psi_{-}(x)=C{\overline{\psi_{-}(x)}}^{T}
$$

identically, in the sense that these conditions are satisfied regardless of the choice of $N_{R}(x)$ or $N_{L}(x)$; one may replace $N_{R}(x)$ with an arbitrary fermion field $f_{R}(x)$ in Eq. (23), for example, and the resulting $\psi_{+}(x)$ will still satisfy the condition (24). We take the relations (24) combined with the Dirac equations

$$
\left(i \gamma^{\mu} \partial_{\mu}-M_{1}\right) \psi_{+}(x)=0, \quad\left(i \gamma^{\mu} \partial_{\mu}-M_{2}\right) \psi_{-}(x)=0
$$

as the definition of Majorana fermions based on an analysis of the Clifford algebra. One may try to define the Majorana fermion defined in Eq. (23) using a nontrivial charge conjugation operator, but such an attempt generally fails [31]. See, however, Sec. II C and Appendix A.

\footnotetext{
${ }^{3}$ The relation (19) may be regarded as the ordinary AutonneTakagi factorization of the matrix $(-i)\left(\begin{array}{cc}m_{R}^{\dagger} & m_{D} \\ m_{D} & m_{L}\end{array}\right)$. Also, the relation (19) written in terms of a unitary matrix $U e^{-i \pi / 4}$ will be discussed in Appendix A in connection with the Pauli-Gürsey transformation. Mathematically, the Autonne-Takagi factorization with a suitable unitary matrix gives rise to characteristic values. Thus $M_{1}$ and $M_{2}$ can be chosen to be real and positive for a suitable $U e^{-i \pi / 4}$ in Eq. (19). A crucial difference between the Autonne-Takagi factorization and the conventional unitary transformation is that one can change the phase of the diagonal elements freely by choosing a suitable $U$ as in Eq. (19).
}

The transformation (20) preserves the form of the kinetic term in the Lagrangian and thus the canonical anticommutators; the transformation thus constitutes a canonical transformation. The discrete symmetry rules (14) are thus applied to new variables every time after the canonical transformation [32-34]. ${ }^{4}$ As explained in Appendix A, the $U(2)$ transformation (20) is related to the Pauli-Gürsey transformation.

The Lagrangian (22) is not invariant under the $C$ or $P$ transformation defined conventionally in Eq. (14) separately for $M_{1} \neq M_{2}$, as shown below, while the Lagrangian is invariant under the $C P$ transformation in Eq. (14), $N_{L}(x) \rightarrow-\gamma^{0} C{\overline{N_{L}(t,-\vec{x}}}^{T}$ and $N_{R}(x) \rightarrow-\gamma^{0} C{\overline{N_{R}(t,-\vec{x})}}^{T}$, for any $M_{1}$ and $M_{2}$. We can also confirm this by using the formal operator notations for the transformations (14)

$$
\begin{aligned}
& (\mathcal{P C})\left\{e^{i \pi / 4} N_{R}(x)-e^{-i \pi / 4} C{\overline{N_{R}(x)}}^{T}\right\}(\mathcal{P C})^{\dagger}=i \gamma^{0} \psi_{+}(t,-\vec{x}), \\
& \left.(\mathcal{P C})\left\{e^{i \pi / 4} N_{L}(x)+e^{-i \pi / 4} C \overline{N_{L}(x)}\right)^{T}\right\}(\mathcal{P C})^{\dagger}=-i \gamma^{0} \psi_{-}(t,-\vec{x}) .
\end{aligned}
$$

We thus characterize these Majorana fields by $C P$ symmetry in Eq. (14),

$$
\begin{aligned}
& \left.(\mathcal{P C}) \psi_{+}(x)(\mathcal{P C})^{\dagger}=-i \gamma^{0} C \overline{\psi_{+}(t,-\vec{x}}\right)^{T}=i \gamma^{0} \psi_{+}(t,-\vec{x}), \\
& \left.(\mathcal{P C}) \psi_{-}(x)(\mathcal{P C})^{\dagger}=-i \gamma^{0} C \overline{\psi_{-}(t,-\vec{x}}\right)^{T}=-i \gamma^{0} \psi_{-}(t,-\vec{x}),
\end{aligned}
$$

\footnotetext{
${ }^{4}$ We also apply the same transformation rules to the old variables. Thus the $C, P$, and $C P$ transformations of the old variables do not reproduce in general those symmetries of the new variables after the canonical transformation, which changes the forms of the mass terms and interaction terms. The classic Kobayashi-Maskawa analysis of $C P$ violation illustrates an example of the use of a canonical transformation [35].
} 
where the first equalities are the operator relations and the second equalities are the classical Majorana conditions (24); combined, these two operations reproduce Eq. (26). It is crucial that these $C P$ transforms are consistent with the classical Majorana conditions in the sense that

$$
\begin{aligned}
& \psi_{+}(x)=-C{\overline{\psi_{+}(x)}}^{T} \rightarrow i \gamma^{0} \psi_{+}(t,-\vec{x})=-C \overline{i \gamma^{0} \psi_{+}(t,-\vec{x})}, \\
& \psi_{-}(x)=C{\overline{\psi_{-}(x)}}^{T} \rightarrow-i \gamma^{0} \psi_{-}(t,-\vec{x})=C \overline{-i \gamma^{0} \psi_{-}(t,-\vec{x})},
\end{aligned}
$$

which is a counterpart of the crucial consistency of the $i \gamma^{0}$ parity (9) and the classical Majorana condition (11). In this formulation, we do not specify the parity transformation for the Majorana field, but rather the $C P$ parity. One can see that, while the $C P$ transformation (27) is specified and leaves the Lagrangian invariant, we have for the $\gamma_{0}$-parity

$$
\begin{aligned}
\mathcal{P} \psi_{+}(x) \mathcal{P}^{\dagger} & =\mathcal{P}\left\{e^{i \pi / 4} N_{R}(x)-e^{-i \pi / 4} C \overline{N_{R}(x)^{T}}\right\} \mathcal{P}^{\dagger} \\
& =e^{i \pi / 4} \gamma^{0} N_{L}(t,-\vec{x})-e^{-i \pi / 4} C \bar{\gamma}^{0} N_{L}(t,-\vec{x})^{T} \\
& =\gamma^{0}\left[e^{i \pi / 4} N_{L}(t,-\vec{x})+e^{-i \pi / 4} C \overline{N_{L}(t,-\vec{x})^{T}}\right] \\
& =\gamma^{0} \psi_{-}(t,-\vec{x}), \\
\mathcal{P} \psi_{-}(x) \mathcal{P}^{\dagger} & =\gamma^{0} \psi_{+}(t,-\vec{x}),
\end{aligned}
$$

which is not a symmetry of the Lagrangian (22) for $M_{1} \neq M_{2}$, which is required for neutron oscillations.

We emphasize that the relations (29) correspond to the "parity doubling theorem" that we discussed beforenamely, the $\gamma^{0}$-parity invariance of the Lagrangian after mass diagonalization leads to degeneracy of the emergent Majorana fermions $M_{1}=M_{2}$ and thus to no neutronantineutron oscillations [18]. In this sense, $\gamma^{0}$-parity is a criterion for discrimination between the Lagrangians which may lead to oscillations and those that do not: $\gamma^{0}$-parity violation of the baryon-number-violating Lagrangian written in terms of the original neutron field is a necessary condition for oscillation [see Eq. (42)]. Note that this is a technical criterion and that it does not indicate a physically observable parity violation in oscillation, as explained in [18].

The charge conjugation is not a symmetry either:

$$
\begin{aligned}
\mathcal{C} \psi_{+}(x) \mathcal{C}^{\dagger} & =\mathcal{C}\left\{e^{i \pi / 4} N_{R}(x)-e^{-i \pi / 4} C \overline{N_{R}(x)}{ }^{T}\right\} \mathcal{C}^{\dagger} \\
& =e^{i \pi / 4} C{\overline{N_{L}}}^{T}(x)-e^{-i \pi / 4} N_{L}(x) \\
& =i\left[e^{i \pi / 4} N_{L}(x)+e^{-i \pi / 4} C \overline{N_{L}(x)}{ }^{T}\right] \\
& =i \psi_{-}(x), \\
\mathcal{C} \psi_{-}(x) \mathcal{C}^{\dagger} & =-i \psi_{+}(x) .
\end{aligned}
$$

Namely, it is not the symmetry of the Lagrangian (22) for $M_{1} \neq M_{2}$.

\section{B. Neutron-antineutron oscillations}

The starting neutron field, which is understood to be the neutron produced by strong interactions, is related to the "mass eigenstate" $N(x)$ by Eq. (20) that is in turn expressed in terms of Majorana fields. We define the new fields

$$
\hat{n}(x) \equiv e^{i \pi / 4} n(x), \quad \hat{N}(x) \equiv e^{i \pi / 4} N(x)
$$

in terms of which the Majorana fields [23] are written as

$$
\begin{aligned}
& \psi_{+}(x)=\hat{N}_{R}(x)-C{\overline{\hat{N}_{R}(x)}}^{T}, \\
& \psi_{-}(x)=\hat{N}_{L}(x)+C \overline{\hat{N}}_{L}(x)^{T},
\end{aligned}
$$

and we have

$$
\begin{aligned}
\hat{n}(x)= & \hat{n}_{R}+\hat{n}_{L} \\
= & \left(\hat{U}_{11}^{\star} \psi_{+}(x)_{R}-\hat{U}_{21} \psi_{+}(x)_{L}\right) \\
& +\left(\hat{U}_{12}^{\star} \psi_{-}(x)_{R}+\hat{U}_{22} \psi_{-}(x)_{L}\right), \\
\hat{n}^{c}(x)= & \hat{n}_{R}^{c}+\hat{n}_{L}^{c} \\
= & \left(\hat{U}_{21}^{\star} \psi_{+}(x)_{R}-\hat{U}_{11} \psi_{+}(x)_{L}\right) \\
& +\left(\hat{U}_{22}^{\star} \psi_{-}(x)_{R}+\hat{U}_{12} \psi_{-}(x)_{L}\right) .
\end{aligned}
$$

We defined the matrix elements of a new $2 \times 2$ unitary matrix

$$
\begin{aligned}
\hat{U} & \equiv\left(\begin{array}{cc}
e^{-i \pi / 4} & 0 \\
0 & e^{i \pi / 4}
\end{array}\right) U\left(\begin{array}{cc}
e^{i \pi / 4} & 0 \\
0 & e^{-i \pi / 4}
\end{array}\right) \\
& =\left(\begin{array}{cc}
e^{-i \pi / 4} & 0 \\
0 & e^{i \pi / 4}
\end{array}\right)\left(\begin{array}{ll}
U_{11} & U_{12} \\
U_{21} & U_{22}
\end{array}\right)\left(\begin{array}{cc}
e^{i \pi / 4} & 0 \\
0 & e^{-i \pi / 4}
\end{array}\right),
\end{aligned}
$$

which satisfies, instead of Eq. (20),

$$
\left(\begin{array}{l}
\hat{n}_{L}^{c} \\
\hat{n}_{L}
\end{array}\right)=\hat{U}\left(\begin{array}{l}
\hat{N}_{L}^{c} \\
\hat{N}_{L}
\end{array}\right), \quad\left(\begin{array}{l}
\hat{n}_{R} \\
\hat{n}_{R}^{c}
\end{array}\right)=\hat{U}^{\star}\left(\begin{array}{l}
\hat{N}_{R} \\
\hat{N}_{R}^{c}
\end{array}\right) .
$$

The external fields $\hat{n}(x)$ and $\hat{n}^{c}(x)$ are treated as analogs of "flavor" fields in these neutron-antineutron oscillations. ${ }^{5}$ When one uses the (valid) $C P$ symmetry of Majorana fermions in Eq. (27), it is confirmed that the relations (33) show that $C P$ symmetry is broken for $\hat{U} \neq \hat{U}^{\star}$ in the sense that

\footnotetext{
${ }^{5}$ If one adjusts the phase conventions of the starting neutron fields $n(x)$ and $n^{c}(x)$ suitably in Eq. (1), one can avoid the use of fields with the hat notation. We prefer, however, to keep the present notational convention to emphasize that we start with a generic Lagrangian (1) and examine what happens if one applies the conventional $\gamma^{0}$ parity operation.
} 


$$
\mathcal{C} P \hat{n}(x)(C P)^{\dagger} \neq-i \gamma^{0} \hat{n}^{c}(t,-\vec{x}) .
$$

Namely, the operations of $C P$ at the level of Majorana fermions do not agree with the expected operations of $C P$ at the level of the neutron $\hat{n}(x)$ produced by strong interactions [using the definition of Eq. (31) and the transformation rules (14)].

The unitary matrix $U$ in Eq. (20) [or the matrix $\hat{U}$ (34) among the variables with a hat] transfers the $C P$-violating effects to the interaction terms, which contains the coupling to other particles such as the proton depending on the detailed specification of the effective model, leaving the $C P$ invariant Lagrangian (22) for the sector of Majorana fermions. Unlike the $C P$ analysis of the seesaw model in an extension of the Standard Model [31,36-38], which is described by a Lagrangian closely related to Eq. (12), this effective theory is not designed to analyze the $C P$ symmetry breaking, since the weak current which describes the transition between $n(x)$ and $p(x)$ is not purely left-handed and $n_{L}$ is a superposition of mass eigenstates $N_{L}$ and $N_{L}^{c}$ with approximately equal weight factors. This case is very different from the Standard Model. A realistic analysis of $C P$ symmetry breaking related to the transition between $n(x)$ and $p(x)$ would require a study of the fundamental quark level dynamics.

On the other hand, this effective theory is useful for understanding a general qualitative aspect of $C P$ symmetry such as the question of whether $C P$ symmetry can be measured in neutron-antineutron oscillations by treating $n(x)$ and $n^{c}(x)$ as analogs of flavor fields. We discuss this aspect of $C P$ symmetry here.

As for the neutron-antineutron oscillations, assuming a sudden projection treatment ("sudden" in the sense of the change of the description in terms of a neutron to the description in terms of nondegenerate Majorana fermions), we have, by defining the neutron state at $t=0$ as $|n(0, \vec{p})\rangle=\hat{n}^{\dagger}(0, \vec{p})^{T}|0\rangle$ and similarly the antineutron state at the time $t$ as $\langle\bar{n}(t, \vec{p})|=\langle 0| \hat{n}^{c}(t, \vec{p})^{T}$,

$$
\begin{aligned}
\langle\bar{n}(t, \vec{p}) \mid n(0, \vec{p})\rangle= & \left.\left(\hat{U}_{21}^{\star} \hat{U}_{11}\right)\left[\left\langle\psi_{+R}(t, \vec{p}) \mid \psi_{+R}(0, \vec{p})\right\rangle+\left\langle\psi_{+L}(t, \vec{p})\right| \psi_{+L}(0, \vec{p})\right]\right\rangle \\
& +\left(\hat{U}_{22}^{\star} \hat{U}_{12}\right)\left[\left\langle\psi_{-R}(t, \vec{p}) \mid \psi_{-R}(0, \vec{p})\right\rangle+\left\langle\psi_{-L}(t, \vec{p}) \mid \psi_{-L}(0, \vec{p})\right\rangle\right] \\
= & \left(\hat{U}_{21}^{\star} \hat{U}_{11}\right)\left\langle\psi_{+}(t, \vec{p}) \mid \psi_{+}(0, \vec{p})\right\rangle+\left(\hat{U}_{22}^{\star} \hat{U}_{12}\right)\left\langle\psi_{-}(t, \vec{p}) \mid \psi_{-}(0, \vec{p})\right\rangle .
\end{aligned}
$$

If one notes the relation $\hat{U}_{21} \hat{U}_{11}^{\star}+\hat{U}_{22} \hat{U}_{12}^{\star}=0$ arising from the unitarity of $\hat{U}$, one obtains

$$
\begin{aligned}
|\langle\bar{n}(t, \vec{p}) \mid n(0, \vec{p})\rangle|^{2} & =\left|\left(\hat{U}_{21} \hat{U}_{11}^{\star}\right)\right|^{2}\left|\left[\left\langle\psi_{+}(t, \vec{p}) \mid \psi_{+}(0, \vec{p})\right\rangle-\left\langle\psi_{-}(t, \vec{p}) \mid \psi_{-}(0, \vec{p})\right\rangle\right]\right|^{2} \\
& =\left|\left(\hat{U}_{21} \hat{U}_{11}^{\star}\right)\right|^{2}\left|\left[e^{i E_{1} t}\left\langle\psi_{+}(0, \vec{p}) \mid \psi_{+}(0, \vec{p})\right\rangle-e^{i E_{2} t}\left\langle\psi_{-}(0, \vec{p}) \mid \psi_{-}(0, \vec{p})\right\rangle\right]\right|^{2} \\
& =4\left|\left(\hat{U}_{21} \hat{U}_{11}^{\star}\right)\right|^{2} \mid \sin ^{2}(\Delta E t / 2),
\end{aligned}
$$

where $\Delta E=E_{1}-E_{2}$, with $E_{i}=\sqrt{\vec{p}^{2}+M_{i}^{2}}, i=1,2$, and where we use $\left\langle\psi_{+}(0, \vec{p}) \mid \psi_{+}(0, \vec{p})\right\rangle=\left\langle\psi_{-}(0, \vec{p}) \times\right.$ $\left|\psi_{-}(0, \vec{p})\right\rangle=1$. It is significant that the amplitude $\langle\bar{n}(t, \vec{p}) \mid n(0, \vec{p})\rangle$ is expressed in terms of the well-defined $\left\langle\psi_{+}(t, \vec{p}) \mid \psi_{+}(0, \vec{p})\right\rangle \quad$ and $\left\langle\psi_{-}(t, \vec{p}) \mid \psi_{-}(0, \vec{p})\right\rangle \quad$ without any chiral projection operators in this treatment. Hence, the use of chiral fermions is a matter of mathematical convenience.

Equation (38) shows that the effect of $C P$ breaking does not appear in the oscillation probability in this effective theory, although the absolute values of the amplitude depend on the possible $C P$ breaking, which is in agreement with the conclusion drawn in [18]. This has been confirmed by a detailed calculation using a quantum field theoretical procedure using a method of unitarily inequivalent representations in the Hamiltonian formalism [20].

For the sake of completeness, we present here an exact mass difference after the mass diagonalization (19). We first rewrite Eq. (19) in the form
$U^{\dagger}\left(\begin{array}{cc}m_{D}^{2}+\left|m_{R}\right|^{2} & m_{D}\left(m_{R}+m_{L}\right) \\ m_{D}\left(m_{R}^{\dagger}+m_{L}^{\dagger}\right) & m_{D}^{2}+\left|m_{L}\right|^{2}\end{array}\right) U=\left(\begin{array}{cc}M_{1}^{2} & 0 \\ 0 & M_{2}^{2}\end{array}\right)$.

From the considerations of the trace and the determinant of this relation, we have

$$
\begin{aligned}
M_{1}^{2}+M_{2}^{2} & =2 m_{D}^{2}+\left|m_{R}\right|^{2}+\left|m_{L}\right|^{2}, \\
M_{1}^{2} M_{2}^{2} & =\left(m_{D}^{2}+\left|m_{R}\right|^{2}\right)\left(m_{D}^{2}+\left|m_{L}\right|^{2}\right)-m_{D}^{2}\left|m_{R}+m_{L}\right|^{2},
\end{aligned}
$$

and thus

$$
\begin{aligned}
\left(M_{1}^{2}-M_{2}^{2}\right)^{2} & =4 m_{D}^{2}\left|m_{R}+m_{L}\right|^{2}+\left(\left|m_{R}\right|^{2}-\left|m_{L}\right|^{2}\right)^{2} \\
& =16 m_{D}^{2}|m|^{2}+4\left(m m_{5}^{\star}+m^{\star} m_{5}\right)^{2},
\end{aligned}
$$

which implies $\left|M_{1}^{2}-M_{2}^{2}\right|=4 m_{D}|m|$ for $m_{D} \gg\left|m_{5}\right|$ for an arbitrary $m \neq 0$. Finally, 


$$
\left|M_{1}-M_{2}\right|=2|m|
$$

for $m_{D} \gg|m|$. In the same approximation, one has $M_{1,2}=$ $m_{D} \pm|m|$ when choosing $M_{1}>M_{2}$.

The complete absence of $C P$ breaking implies $\hat{U}=\hat{U}^{\star}$ in Eq. (36) —namely, one may choose a real unitary $\hat{U}$ in Eq. (34) which is a generic orthogonal matrix:

$$
\hat{U}=\left(\begin{array}{cc}
\cos \theta & \sin \theta \\
-\sin \theta & \cos \theta
\end{array}\right)
$$

Thus in the absence of the $C P$ violation contained in $\hat{U}$, we have the standard formula for the neutron-antineutron oscillation probability [for nonrelativistic neutrons with $m_{D} \gg|m|$ as in Eq. (42)]:

$$
\left|\left\langle\bar{n}_{0}(t, \vec{p}) \mid n_{0}(0, \vec{p})\right\rangle\right|^{2}=\sin ^{2}(2 \theta) \sin ^{2}(\Delta E t / 2) .
$$

\section{Deformed symmetry generated by $\mathcal{C}_{M}$ and $\mathcal{P}_{M}$}

One may wonder whether it is possible to define $C$ and $P$ symmetries valid for the emergent Majorana fermions in this formulation. It is possible to define a formal deformed symmetry generated by $[27,34]$

$$
\mathcal{C}_{M}=1, \quad \mathcal{P}_{M}=\mathcal{P C},
$$

which is a symmetry of Eq. (22) and

$$
\begin{array}{ll}
\mathcal{C}_{M} \psi_{+}(x) \mathcal{C}_{M}^{\dagger}=\psi_{+}(x), & \mathcal{P}_{M} \psi_{+}(x) \mathcal{P}_{M}^{\dagger}=i \gamma^{0} \psi_{+}(t,-\vec{x}), \\
\mathcal{C}_{M} \psi_{-}(x) \mathcal{C}_{M}^{\dagger}=\psi_{-}(x), & \mathcal{P}_{M} \psi_{-}(x) \mathcal{P}_{M}^{\dagger}=-i \gamma^{0} \psi_{-}(t,-\vec{x}),
\end{array}
$$

as given in Eq. (27). The nontrivial part of this deformation is the $C P$ symmetry and, in this sense, this deformation is essentially equivalent to the formulation of the Majorana fermion with $P C=\mathcal{P}_{M} \mathcal{C}_{M}$ as described above. It is assuring that the "parity" defined in Eq. (46) corresponds to $\pm i \gamma^{0}$-parity and is thus consistent with the classical Majorana condition. The classical Majorana condition $\psi_{+}(x)=-C{\overline{\psi_{+}(x)}}^{T}$ or $\psi_{-}(x)=C{\overline{\psi_{-}(x)}}^{T}$ in Eq. (24), which determines whether a given fermionic field is a Majorana field, carries the same physical information as the trivial operation $\mathcal{C}_{M} \psi_{ \pm}(x) \mathcal{C}_{M}^{\dagger}=\psi_{ \pm}(x)$ applied to the field $\psi_{ \pm}(x)$, which is assumed to be the Majorana fermion $\left.\psi_{+}(x)=-C \overline{\psi_{+}(x)}\right)^{T}$ or $\left.\psi_{-}(x)=C \overline{\psi_{-}(x)}\right)^{T}$, respectively. Physicswise, those modified $C$ and $P$ symmetries do not add new ingredients to the analysis of neutron-antineutron oscillations.

\section{DISCUSSION AND CONCLUSION}

We have shown that the use of the conventional $\gamma^{0}$-parity for the starting neutron field gives rise to a consistent description of the emergent Majorana fermions in the oscillation process and thus a consistent description of neutron-antineutron oscillations. Physically, this choice is warranted by the fact that the neutron produced in strong interactions is viewed as a Dirac particle, with the oscillation-inducing Majorana mass terms being the effective expression of some (thus far hypothetical) additional interaction. The crucial observation is that the emergent Majorana fermions are characterized by $C P$ symmetry and are consistent with the classical Majorana condition, as in Eqs. (27) and (28). Technically, the $C$ and $P$ defined for the starting neutron are not good symmetries of the emergent Majorana fermions in the chiral description (22) for $M_{1} \neq M_{2}$, which is required for neutron-antineutron oscillations, and thus the choice of $\gamma^{0}$-parity or $i \gamma^{0}$-parity for the initial neutron does not make a decisive difference (see also Appendix A).

Although the general Lagrangian (1) is physically $P$ and $C P$ violating, the definitions of $\gamma^{0}$ - or $i \gamma^{0}$-parities still have their respective merits in the analysis of neutron oscillations: the $\gamma^{0}$-parity produces a criterion, in the form of the parity doubling theorem, for the existence of oscillations for particular choices of the mass parameters in Eq. (1) (see Ref. [18]), while $i \gamma^{0}$-parity emphasizes the $P$ and $C P$ invariance of the oscillation probability (see Refs. [18,21]).

This formulation supports past analyses of neutronantineutron oscillations using the ordinary $\gamma^{0}$-parity conducted, for example, in $[15,18]$ from a different theoretical perspective.

\section{ACKNOWLEDGMENTS}

We thank M. Chaichian for helpful comments. This work is supported in part by JSPS KAKENHI (Grant No. 18K03633).

\section{APPENDIX A: PAULI-GÜRSEY TRANSFORMATION}

We show that the different choice of the parity operation, $i \gamma^{0}$ or $\gamma^{0}$, is compensated for by the Pauli-Gürsey transformation [32-34] in the diagonalization process of the mass matrix. We thus formally understand the canonical equivalence of the two choices of parity operation in an analysis of neutron-antineutron oscillations on the basis of an effective Lagrangian (1).

In the formulation with $i \gamma^{0}$-parity as given in [21], one may choose the Autonne-Takagi factorization of a complex symmetric matrix [instead of Eq. (19)]

$$
U^{\prime T}\left(\begin{array}{ll}
m_{R}^{\dagger} & m_{D} \\
m_{D} & m_{L}
\end{array}\right) U^{\prime}=\left(\begin{array}{cc}
M_{1} & 0 \\
0 & -M_{2}
\end{array}\right)
$$

and define 


$$
\left(\begin{array}{c}
n_{L}^{c} \\
n_{L}
\end{array}\right)=U^{\prime}\left(\begin{array}{c}
N_{L}^{c} \\
N_{L}
\end{array}\right), \quad\left(\begin{array}{c}
n_{R} \\
n_{R}^{c}
\end{array}\right)=U^{\prime \star}\left(\begin{array}{c}
N_{R} \\
N_{R}^{c}
\end{array}\right) .
$$

Note that the Autonne-Takagi factorization is very different from the conventional diagonalization of a Hermitian matrix by a unitary transformation: the Autonne-Takagi factorization basically gives rise to characteristic values (i.e., real and positive $M_{1}$ and $M_{2}$ ), but the phase freedom of the diagonal elements is still left free. The total Hermitian Lagrangian (12) is then written as

$$
\begin{aligned}
\mathcal{L}= & \frac{1}{2}\left\{\overline{N_{L}}(x) i \not \partial N_{L}(x)+\overline{N_{L}^{c}}(x) i \not \partial N_{L}^{c}(x)+\overline{N_{R}}(x) i \not \partial N_{R}(x)+\overline{N_{R}^{c}}(x) i \not \partial N_{R}^{c}(x)\right\} \\
& -(1 / 2)\left(\overline{N_{R}} \quad \overline{N_{R}^{c}}\right)\left(\begin{array}{cc}
M_{1} & 0 \\
0 & -M_{2}
\end{array}\right)\left(\begin{array}{c}
N_{L}^{c} \\
N_{L}
\end{array}\right)+\text { H.c. } \\
= & \overline{N_{L}}(x) i \not \partial N_{L}(x)+\overline{N_{R}}(x) i \not \partial N_{R}(x)-(1 / 2)\left\{\overline{N_{R}} C M_{1} \overline{N_{R}}{ }^{T}+N_{R}^{T} C M_{1} N_{R}-\overline{N_{L}} C M_{2} \overline{N_{L}}{ }^{T}-N_{L}^{T} C M_{2} N_{L}\right\} \\
= & \frac{1}{2} \overline{\psi_{+}(x)}\left(i \gamma^{\mu} \partial_{\mu}-M_{1}\right) \psi_{+}(x)+\frac{1}{2} \overline{\psi_{-}(x)}\left(i \gamma^{\mu} \partial_{\mu}-M_{2}\right) \psi_{-}(x),
\end{aligned}
$$

where we define the Majorana fields by

$$
\begin{aligned}
& \psi_{+}(x)=N_{R}(x)+C{\overline{N_{R}(x)}}^{T}, \\
& \psi_{-}(x)=N_{L}(x)-C{\overline{N_{L}(x)}}^{T},
\end{aligned}
$$

and they satisfy the classical Majorana conditions

$$
\psi_{+}(x)=C{\overline{\psi_{+}(x)}}^{T}, \quad \psi_{-}(x)=-C{\overline{\psi_{-}(x)}}^{T}
$$

identically in the sense that these conditions are satisfied regardless of the choice of $N_{R}(x)$ or $N_{L}(x)$. The Lagrangian (A3) is invariant under the $C P$ symmetry

$$
N_{L}(x) \rightarrow i \gamma^{0} C{\overline{N_{L}(t,-\vec{x})}}^{T}, \quad N_{R}(x) \rightarrow i \gamma^{0} C{\overline{N_{R}(t,-\vec{x})}}^{T}
$$

defined by $i \gamma^{0}$ parity for any real $M_{1}$ and $M_{2}$, and the same $C P$ gives

$$
\begin{aligned}
& (\mathcal{P C}) \psi_{+}(x)(\mathcal{P C})^{\dagger}=i \gamma^{0} \psi_{+}(t,-\vec{x}), \\
& (\mathcal{P C}) \psi_{-}(x)(\mathcal{P C})^{\dagger}=-i \gamma^{0} \psi_{-}(t,-\vec{x}),
\end{aligned}
$$

which are consistent with the classical Majorana conditions (A5). According to the $i \gamma_{0}$ modification of the transformations (14) which treat $N_{L}$ and $N_{R}$ as the chiral components of a Dirac field, we note, however, that neither $C$

$$
\begin{aligned}
& \mathcal{C} \psi_{+}(x) \mathcal{C}^{\dagger}=N_{L}(x)+C{\overline{N_{L}(x)^{T}}}^{T} \\
& \mathcal{C} \psi_{-}(x) \mathcal{C}^{\dagger}=-N_{R}(x)+C{\overline{N_{R}(x)}}^{T}
\end{aligned}
$$

nor $P\left(i \gamma^{0}\right.$-parity)

$$
\begin{aligned}
& \mathcal{P} \psi_{+}(x) \mathcal{P}^{\dagger}=i \gamma^{0}\left[N_{L}(x)+C{\overline{N_{L}(x)}}^{T}\right], \\
& \mathcal{P} \psi_{-}(x) \mathcal{P}^{\dagger}=i \gamma^{0}\left[N_{R}(x)-C{\overline{N_{R}(x)}}^{T}\right]
\end{aligned}
$$

are good symmetries of Eq. (A3) for $M_{1} \neq M_{2}$ [i.e., $|m| \neq 0$ in Eq. (42) using a fact mentioned below]. Nevertheless the Lagrangian (A3) is physically $P$ and $C$ invariant, upon a redefinition of these transformations which complies with the Majorana condition (A4), using the deformed symmetry generators as in Eq. (45).

When one remembers that the starting mass matrix and the neutron field are common and that the mass eigenvalues $M_{1}$ and $M_{2}$ are common for either choice of the parity operation, as is directly confirmed by deriving the relations (39) and (40) for the relation (A1) also with $U \rightarrow U^{\prime}$, one can confirm that the matrix $U^{\prime}$ in Eq. (A1) is written using the $U$ in Eq. (19) as

$$
U^{\prime}=U U_{0}
$$

with

$$
U_{0}=e^{-i \pi / 4}\left(\begin{array}{ll}
1 & 0 \\
0 & i
\end{array}\right)=\left(\begin{array}{cc}
e^{-i \pi / 4} & 0 \\
0 & e^{i \pi / 4}
\end{array}\right)
$$

The mass eigenstates in Eq. (A2) and the mass eigenstates in Eq. (20) are then related by

$U_{0}\left(\begin{array}{l}N_{L}^{c} \\ N_{L}\end{array}\right)_{i \gamma^{0}}=\left(\begin{array}{c}N_{L}^{c} \\ N_{L}\end{array}\right)_{\gamma^{0}}, \quad U_{0}^{\star}\left(\begin{array}{c}N_{R} \\ N_{R}^{c}\end{array}\right)_{i \gamma^{0}}=\left(\begin{array}{c}N_{R} \\ N_{R}^{c}\end{array}\right)_{\gamma^{0}}$,

with $U_{0} \in U(2)$ of the Pauli-Gürsey canonical transformation [32-34]. In this sense, the two different definitions of parity are canonically equivalent. The secret of the appearance of this relation is traced to the hidden freedom in the definition of classical Majorana fermions; the definition of Majorana fermions in Eq. (A4) is extended to the definition of Majorana fermions in Eq. (23) with a phase freedom which is precisely related to this freedom of the canonical transformation. To be precise, it is confirmed that 


$$
\left\{\psi_{ \pm}(x)\right\}_{i \gamma^{0}}=(-i)\left\{\psi_{ \pm}(x)\right\}_{\gamma^{0}}
$$

in this phase convention, which is consistent with the classical Majorana conditions (24) and (A5). It is confirmed that the same oscillation formula as in Eq. (38) is valid for the description with $i \gamma^{0}$-parity by noting that $\left|\left(\hat{U}_{21} \hat{U}_{11}^{\star}\right)\right|^{2}=$ $\left|\left(U_{21} U_{11}^{\star}\right)\right|^{2}=\left|\left(U_{21}^{\prime} U_{11}^{\prime \star}\right)\right|^{2}$. The $C P$ invariance corresponds to $U^{\prime}=U^{\prime \star}$.

Finally, we would like to add a comment on the formulation in [21] from our point of view. We recall the use of a specific Pauli-Gürsey transformation in the context of a seesaw model of neutrinos [26], which is relevant to this problem. As for a related use of the Bogoliubov transformation; see [31,39]. One may apply the Pauli-Gürsey $U(2)$ transformation

$$
\left(\begin{array}{c}
N_{L}^{c} \\
N_{L}
\end{array}\right)=O\left(\begin{array}{c}
\tilde{n}_{L}^{c} \\
\tilde{n}_{L}
\end{array}\right), \quad\left(\begin{array}{c}
N_{R} \\
N_{R}^{c}
\end{array}\right)=O\left(\begin{array}{c}
\tilde{n}_{R} \\
\tilde{n}_{R}^{c}
\end{array}\right)
$$

with an element

$$
O=\frac{1}{\sqrt{2}}\left(\begin{array}{cc}
1 & 1 \\
-1 & 1
\end{array}\right)
$$

to the first line of the Lagrangian (A3), which corresponds to a single generation model of the neutrino. One obtains

$$
\begin{aligned}
\mathcal{L}= & 1 / 2\left\{\overline{\tilde{n}}(x) i \not \partial \tilde{n}(x)+\overline{\tilde{n}^{c}}(x) i \not \partial \tilde{n}^{c}(x)\right\} \\
& -1 / 4\left\{\tilde{\tilde{n}}\left(M_{1}+M_{2}\right) \tilde{n}+\overline{\tilde{n}^{c}}\left(M_{1}+M_{2}\right) \tilde{n}^{c}\right\} \\
& -1 / 4\left\{\tilde{\tilde{n}}\left(M_{1}-M_{2}\right) \tilde{n}^{c}+\overline{\tilde{n}^{c}}\left(M_{1}-M_{2}\right) \tilde{n},\right\},
\end{aligned}
$$

which is invariant under $C, P\left(i \gamma^{0}\right.$-parity), and $C P$. See Eq. (45) and Eqs. (55)-(57) in [26]. If one sets $M_{1}+M_{2}=$ $2 M$ and $M_{1}-M_{2}=2 \epsilon$, this Lagrangian becomes

$$
\mathcal{L}=\overline{\tilde{n}}(x) i \not \partial \tilde{n}(x)-M \overline{\tilde{n}} \tilde{n}-\frac{1}{2} \epsilon\left[\tilde{\tilde{n}} C \overline{\tilde{n}}^{T}+\tilde{n}^{T} C \tilde{n}\right],
$$

which has precisely the form of the Lagrangian discussed in [21].

This shows that our starting Lagrangian (1), our Lagrangians (22) and (A3), and the Lagrangian used in [21] are related by the Pauli-Gürsey transformation and thus canonically equivalent. This fact, however, does not imply that our starting Lagrangian (1) is $C, P$ ( $i \gamma^{0}$-parity), and $C P$ invariant since the Pauli-Gürsey unitary transformations can carry these symmetries to the interaction sector depending on the model. Let us note that, in the context of neutron oscillations, the Lagrangian (A17) does not make the analysis of discrete transformations more transparent than the diagonal Majorana Lagrangian (A3). Moreover, the parameter $M$ in (A17) is not the neutron mass $m_{D}$, but rather a complicated relation between the mass parameters in Eq. (1) (calculated exactly in [18]), making the meaning of $M$ phenomenologically less transparent. There is no reason why one should adopt the Lagrangian (A17), which is one of the canonically equivalent Lagrangians, to describe the neutron-antineutron oscillations.

Incidentally, one can confirm that the $\gamma^{0}$-parity becomes good symmetry for $M_{1}=M_{2}$ in either Eq. (22) or Eq. (A3) [and also in Eq. (A16)] and thus leads to the parity-doublet theorem-namely, no oscillations [18].

\section{APPENDIX B: DIRECT PARITY ANALYSIS OF THE $\Delta B=2$ LAGRANGIAN}

The Lagrangian (1) was systematically analyzed in [18] regarding the physical $P, C$, and $C P$ symmetries before and after diagonalization. Here we give a summary of those results. One can easily take the Lagrangian (1) to the form

$$
\begin{aligned}
\mathcal{L}= & \bar{n}(x) i \gamma^{\mu} \partial_{\mu} n(x)-m_{D} \bar{n}(x) n(x) \\
& -\frac{i}{2}|m|\left[e^{i \alpha} n^{T}(x) C n(x)-e^{-i \alpha} \bar{n}(x) C \bar{n}^{T}(x)\right] \\
& -\frac{i}{2}\left|m_{5}\right|\left[n^{T}(x) C \gamma_{5} n(x)+\bar{n}(x) C \gamma_{5} \bar{n}^{T}(x)\right],
\end{aligned}
$$

where $m_{D}$ and $\alpha$ are real parameters, by a redefinition of the neutron field which absorbs the phase of $m_{5}$. No other redefinitions of the neutron field are permitted due to the baryon number symmetry violation.

One can check directly to ensure that, under the $\gamma_{0}$-parity definition of the neutron field $n$, the $|m|$ term in Eq. (B1) is parity odd, while the $\left|m_{5}\right|$ term is even; under $i \gamma_{0}$-parity, the $|m|$ term in Eq. (B1) is parity even, while the $\left|m_{5}\right|$ term is odd. Actually, any definition of the parity with an arbitrary phase, $e^{i \varphi} \gamma_{0}$-parity, leads to a result of parity violation. The charge conjugation is generally not conserved either. One confirms that, irrespective of the value of $\alpha \neq 0$ in Eq. (B1), the $C P$ violation cannot be eliminated as long as $m, m_{5} \neq 0$. Consequently, for $\alpha \neq 0$ and $m m_{5} \neq 0$ in Eq. (B1), parity and $C P$ are intrinsically violated.

Upon diagonalization, the Lagrangian (B1) is brought to the form of a sum of two free Majorana Lagrangians,

$$
\begin{aligned}
\mathcal{L}= & \frac{1}{2} \overline{\psi_{+}(x)}\left(i \gamma^{\mu} \partial_{\mu}-M_{1}\right) \psi_{+}(x) \\
& +\frac{1}{2} \overline{\psi_{-}(x)}\left(i \gamma^{\mu} \partial_{\mu}-M_{2}\right) \psi_{-}(x),
\end{aligned}
$$

where

$$
\begin{aligned}
M_{1,2}= & \left(\left[\sqrt{m_{D}^{2}+\left|m_{5}\right|^{2}} \pm|m| \sqrt{1-(|\tilde{m}| /|m|)^{2}}\right]^{2}\right. \\
& \left.+(|\tilde{m}|)^{2}\right)^{1 / 2}
\end{aligned}
$$


with $|\tilde{m}| \equiv \epsilon_{1} \sin \alpha \sin 2 \phi$ and $\sin 2 \phi \equiv\left|m_{5}\right| / \sqrt{m_{D}^{2}+\left|m_{5}\right|^{2}}$. On the other hand, the exact solution of the relation (43) is written for the parameterization of mass parameters in (B1) as

$$
M_{1,2}^{2}=m_{D}^{2}+\left|m_{5}\right|^{2}+|m|^{2} \pm 2|m| \sqrt{m_{D}^{2}+\left|m_{5}\right|^{2} \cos ^{2} \alpha} .
$$

One can confirm that (B2) agrees exactly with (B3). This shows that the characteristic values of the mass matrix are invariant under the canonical transformation, and the oscillation formula in [18] is exact and agrees with (38), as it should be.

In terms of the original parameters in the Lagrangian (1), we note that the mass splitting between the Majorana fields, and consequently the oscillation, can occur only if $m \neq 0$. Taking into account that in the physically relevant situation $|m|,\left|m_{5}\right| \ll m_{D}$, one finds from Eq. (B2) in this approximation that $\left|M_{1}-M_{2}\right|=2|m|$ [see also formula (42)] - namely, the correction to the value of the probability of oscillation due to the $C P$-breaking parameters $\alpha$ and $m_{5}$, though not exactly vanishing, is immaterial. However, for any value of the parameters in the Lagrangian (1), the probability of oscillation per se is $P$ and $C P$ invariant. In other words, although the general
Lagrangian is $P$ and $C P$ violating, the oscillation probability is not.

The choice of the conventional $\gamma^{0}$-parity transformation is as legitimate as the choice of the $i \gamma^{0}$-parity transformation advocated as the only sensible choice in Ref. [21], since the general $\Delta B=2$ Lagrangian (1) written in terms of the original neutron field $n(x)$ is not invariant under any of them as long as $m m_{5} \neq 0$, and in general it is not invariant under any conceivable $e^{i \varphi} \gamma_{0}$-parity transformation (see [18]). As a result, the Lagrangian (1) describes effectively mass-generating interactions which break parity, and in general also $C P$ [unless $\alpha$ is set to zero in Eq. (B1) by hand or by physical arguments]. This breaking is not observable in neutron-antineutron oscillations, which are based on the simple quadratic Majorana Lagrangian (22), but it is carried over to the interaction terms by the mixing matrix $U$. Moreover, as shown in [18], if high-energy $C P$-violating interactions generate the $m_{5}$ term in the Lagrangian (1), their effects would show up in an enhanced electric dipole moment of the neutron compared to the one generated by the QCD $\theta$ term. In short, no single choice of the parity transformation of the original neutron field $n(x)$ gives more information than any other when the total Lagrangian, with all the interactions included, is taken into consideration.
[1] V. A. Kuzmin, JETP Lett. 12, 228 (1970).

[2] R. N. Mohapatra and R. E. Marshak, Phys. Rev. Lett. 44, 1316 (1980).

[3] S. L. Glashow, in Proceedings of the 1979 Cargèse Summer Institute on Quarks and Leptons, edited by M. Lévy et al. (Plenum, New York, 1980), p. 687.

[4] L. N. Chang and N. P. Chang, Phys. Lett. 92B, 103 (1980).

[5] R. N. Mohapatra and R. E. Marshak, Phys. Lett. 94B, 183 (1980).

[6] T. K. Kuo and S. Love, Phys. Rev. Lett. 45, 93 (1980).

[7] L. N. Chang and N. P. Chang, Phys. Rev. Lett. 45, 1540 (1980).

[8] K. G. Chetyrkin, M. V. Kazarnovsky, V. A. Kuzmin, and M. E. Shaposhnikov, Phys. Lett. 99B, 358 (1981).

[9] S. Rao and R. E. Shrock, Phys. Lett. 116B, 238 (1982).

[10] R. N. Mohapatra, J. Phys. G 36, 104006 (2009).

[11] D. G. Phillips II et al., Phys. Rep. 612, 1 (2016).

[12] M. Baldo-Ceolin et al. (ILL Collaboration), Z. Phys. C 63, 409 (1994); J. Chung et al. (Soudan II Collaboration), Phys. Rev. D 66, 032004 (2002); K. Abe et al. (SuperKamiokande Collaboration), Phys. Rev. D 91, 072006 (2015); B. Aharmim et al. (SNO Collaboration), Phys. Rev. D 96, 092005 (2017).

[13] A. Addazi et al., arXiv:2006.04907.

[14] Z. Berezhiani and A. Vainshtein, arXiv:1506.05096.
[15] K. Fujikawa and A. Tureanu, arXiv:1510.00868.

[16] D. McKeen and Ann E. Nelson, Phys. Rev. D 94, 076002 (2016).

[17] S. Gardner and Xinshuai Yan, Phys. Rev. D 93, 096008 (2016).

[18] K. Fujikawa and A. Tureanu, Phys. Rev. D 94, 115009 (2016).

[19] K. Fujikawa and A. Tureanu, Phys. Lett. B 777, 240 (2018).

[20] A. Tureanu, Phys. Rev. D 98, 015019 (2018).

[21] Z. Berezhiani and A. Vainshtein, Phys. Lett. B 788, 58 (2019).

[22] J. D. Bjorken and S. D. Drell, Relativistic Quantum Fields (McGraw-Hill, New York, 1965).

[23] E. Majorana, Nuovo Cimento 14, 171 (1937).

[24] B. Kayser, Phys. Rev. D 26, 1662 (1982).

[25] S. Weinberg, The Quantum Theory of Fields I (Cambridge University Press, Cambridge, England, 1995).

[26] K. Fujikawa, Eur. Phys. J. C 80, 285 (2020).

[27] K. Fujikawa, Phys. Rev. D 102, 105001 (2020).

[28] S. Weinberg, Phys. Rev. Lett. 43, 1566 (1979).

[29] L. Autonne, Ann. Univ. Lyon 38, 1 (1915).

[30] T. Takagi, Jpn. J. Math. 1, 83 (1925).

[31] K. Fujikawa and A. Tureanu, Eur. Phys. J. C 79, 752 (2019). 
[32] W. Pauli, Nuovo Cimento 6, 204 (1957).

[33] F. Gürsey, Nuovo Cimento 7, 411 (1958).

[34] K. Fujikawa, Phys. Lett. B 789, 76 (2019).

[35] M. Kobayashi and T. Maskawa, Prog. Theor. Phys. 49, 652 (1973).

[36] M. Fukugita and T. Yanagida, Physics of Neutrinos and Application to Astrophysics (Springer, Berlin, 2002).
[37] C. Giunti and C. W. Kim, Fundamentals of Neutrino Physics and Astrophysics (Oxford University Press, Oxford, 2007).

[38] S. Bilenky, Introduction to the Physics of Massive and Mixed Neutrinos, Lecture Notes in Physics Vol. 817 (Springer, Berlin, 2010).

[39] K. Fujikawa and A. Tureanu, Phys. Lett. B 767, 199 (2017). 